\title{
Nitric oxide directly regulates gene expression during Drosophila development: need some gas to drive into metamorphosis?
}

\author{
Naoki Yamanaka and Michael B. O'Connor ${ }^{1}$ \\ Department of Genetics, Cell Biology, and Development, University of Minnesota, Minneapolis, Minnesota 55455, USA
}

Nitric oxide (NO) is an important second messenger involved in numerous biological processes, but how it regulates gene expression is not well understood. In this issue of Genes \& Development, Cáceres and colleagues (pp. 1476-1485) report a critical requirement of NO as a direct regulator of gene expression through its binding to a heme-containing nuclear receptor in Drosophila. This may be an anciently evolved mechanism to coordinate behavior and metabolism during animal development.

Nitric oxide (NO) is a small diatomic gas that works as a diffusible second messenger in numerous developmental and physiological processes. In insects, its diverse range of functions include regulation of renal fluid production, cell proliferation, synapse formation, and innate immunity (Kuzin et al. 1996; Gibbs and Truman 1998; Kean et al. 2002; Foley and O'Farrell 2003). In many of these cases, NO exerts its function through binding to the heme moiety of soluble guanylate cyclase to stimulate cyclic GMP synthesis. However, the widespread roles of NO in modulating diverse biological processes clearly suggest that this canonical pathway is not unique and that other mechanisms, including protein S-nitrosylation (Hess et al. 2005), exist and need to be further characterized. In 2005, Henry Krause's group (Reinking et al. 2005) reported that NO can bind to the heme-containing Drosophila nuclear receptor E75 in vitro and regulates its interaction with a heterodimer partner, DHR3. This exciting observation, followed by a later report illustrating that the same is true for the mammalian homolog of E75, Rev-erb (Marvin et al. 2009), raised the new possibility that NO might regulate gene expression by directly interacting with a transcription factor. Whether this NO/E75/DHR3 signaling pathway

[Keywords: E75; DHR3; nitric oxide; Drosophila; metamorphosis; ecdysone; metabolism]

${ }^{1}$ Corresponding author.

E-mail moconnor@umn.edu.

Article is online at http://www.genesdev.org/cgi/doi/10.1101/gad.2080411. played a physiologically significant role in vivo, however, remained unknown.

In this issue of Genes \& Development, Krause's research group (Cáceres et al. 2011) now reports a critical function for this signaling pathway in regulating Drosophila steroid production within the major larval endocrine tissue, the prothoracic gland (PG). In almost all cell types, NO is produced by NO synthase (NOS) through the conversion of arginine to citrulline. Cáceres et al. (2011) showed that knockdown of NOS in the PG disrupts the production of ecdysone, the immediate precursor of the steroid molting hormone 20-hydroxyecdysone (20E), leading to metabolic defects and a failure to initiate metamorphosis. Although there is some controversy about the necessity of NOS for Drosophila development and viability (Regulski et al. 2004; Yakubovich et al. 2010), the present study by Cáceres et al. (2011) clearly indicates that NOS is indispensable for Drosophila metamorphosis and plays a critical role in controlling expression of $\beta F T Z$ - $F 1$, a master regulator of insect metamorphosis in many tissues.

\section{Essential role of NO in ecdysteroidogenesis}

Ecdysone biosynthesis (ecdysteroidogenesis) in the PG is mediated by multiple enzymes, each of which is engaged in a specific step of sequential hydroxylation and oxidation reactions (Gilbert et al. 2002). Ecdysone, after being converted into active $20 \mathrm{E}$ in peripheral tissues, triggers gene expression cascades throughout the body leading to developmental transitions; i.e., molting and metamorphosis. Identification and characterization of signaling pathways and transcription factors that regulate expression of ecdysone biosynthetic enzymes, therefore, are critical for a complete understanding of these developmental processes that are central to the insect life cycle.

One such key transcription factor is the nuclear receptor $\beta F T Z-F 1$, an insect homolog of the vertebrate steroidogenic factor 1 that regulates transcription of steroidogenic enzymes. At least two ecdysteroidogenic enzymes are known to be under the control of $\beta F T Z-F 1$ in the Drosophila PG (Parvy et al. 2005). As $\beta F T Z-F 1$ is a direct target 
of DHR3 (Kageyama et al. 1997; Lam et al. 1997), Cáceres et al. (2011) examined whether NO/E75/DHR3 signaling has any role in the regulation of ecdysteroidogenesis. They first confirmed that all of the key players in this pathway (NOS, E75, DHR3, and $\beta$ FTZ-F1) are indeed expressed in the PG when ecdysone synthesis is high (Cáceres et al. 2011). By manipulating the expression levels of NOS, E75, and DHR3 in the PG, they then demonstrated that the interactions between these factors, predicted by their initial in vitro study (Reinking et al. 2005), are conserved in vivo: The DHR3-mediated expression of $\beta F T Z-F 1$ is suppressed by E75, and this inhibitory effect of E75 on $\beta F T Z-F 1$ expression is eliminated by NO produced by NOS (Fig. 1). Notably, when the expression levels of these factors in the PG are manipulated in a way that suppresses $\beta F T Z$-F1 expression, the animals fail to initiate metamorphosis and instead continue to feed and form giant larvae that have high levels of fat accumulation (Cáceres et al. 2011). Since this "huge permanent larva" phenotype is commonly observed in ecdysteroidogenesis-deficient third instar larvae (Berreur et al. 1984; Gibbens et al. 2011) and can be rescued by ecdysone feeding (Cáceres et al. 2011), the present study clearly indicates that NO-mediated E75 inactivation in the PG is necessary to promote ecdysteroidogenesis, which in turn triggers appropriate behavioral

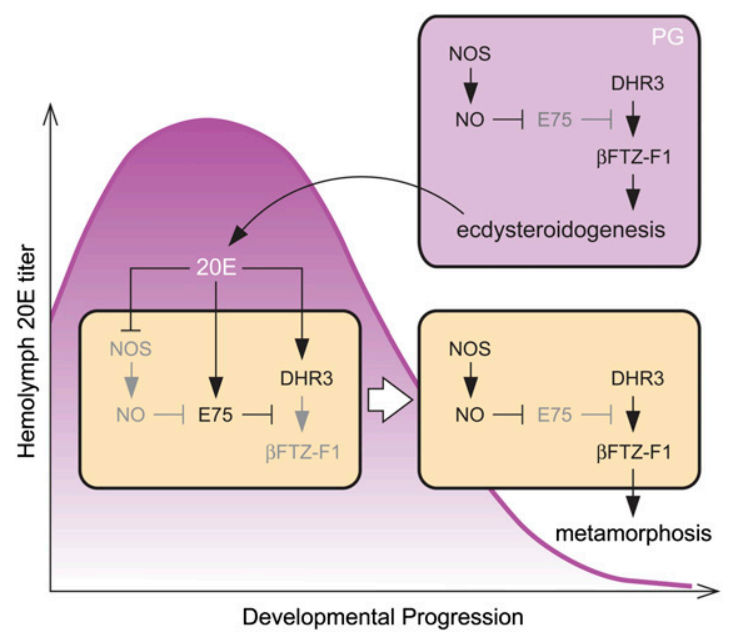

Figure 1. A model for NOS-mediated induction of $\beta F T Z$ - $F 1$ expression during Drosophila metamorphosis. In the PG (pink), NOS-mediated production of NO prevents E75 function as a suppressor of DHR3, allowing DHR3 to induce $\beta F T Z$ - $F 1$ expression. $\beta F T Z-F 1$ in turn promotes ecdysteroidogenesis, at least in part through the induction of ecdysteroidogenic enzymes. In peripheral tissues (yellow), 20E induces expression of both E75 and DHR3. E75 prevents DHR3 function when the hemolymph $20 \mathrm{E}$ titer is high, and 20E-mediated NOS repression through a nongenomic pathway might also be at work to fully potentiate E75 function. Upon decline of the 20E level, E75 quickly degrades to liberate accumulated DHR3, allowing it to induce $\beta F T Z$-F1 expression. Restored NOS function may further block residual E75 to prevent DHR3 function. Transiently expressed $\beta F T Z-F 1$ during midprepupal stage is necessary for the response of peripheral tissues to the next surge of $20 \mathrm{E}$ titer, which promotes metamorphosis throughout the animal body. and metabolic changes (from fat storage to fat utilization) necessary for metamorphosis.

\section{Does NOS regulate $\beta F T Z-F 1$ expression in peripheral tissues?}

Although Cáceres et al. (2011) focused on the PG to demonstrate NOS function in the regulation of $\beta F T Z-F 1$ expression, regulatory mechanisms that control $\beta F T Z$-F1 expression are more extensively studied in peripheral tissues that respond to 20E. Indeed, E75, DHR3, and $\beta F T Z-F 1$ are all transcriptionally regulated by $20 \mathrm{E}$ and show distinct expression profiles in concert with the hemolymph 20E titer fluctuation during development (King-Jones and Thummel 2005). Both E75 and DHR3 are directly induced by the 20E receptor complex EcR/USP, although DHR3 also requires indirect induction for its maximal expression (Segraves and Hogness 1990; Horner et al. 1995). On the other hand, expression of $\beta F T Z-F 1$ is repressed when the $20 \mathrm{E}$ titer is high and is only induced after the hemolymph 20E titer declines (Woodard et al. 1994; Broadus et al. 1999; Yamada et al. 2000; Rewitz et al. 2010). This unique expression profile of $\beta F T Z$-F1 is usually explained by the balance between DHR3 accumulation and E75 decay (White et al. 1997). When the 20E titer is high, one of the E75 isoforms, E75B, suppresses DHR3 activity to induce $\beta F T Z-F 1$. When the 20E titer drops, E75B protein quickly degrades, allowing accumulated DHR3 to induce $\beta F T Z$-F1 expression (Fig. 1). $\beta F T Z-F 1$, being transiently expressed in the midprepupal stage, in turn controls responsiveness of peripheral tissues to the subsequent 20E pulse that triggers overall metamorphic events in the whole body (Woodard et al. 1994; Broadus et al. 1999; Rewitz et al. 2010). $\beta F T Z-F 1$ is thus a crucial competence factor that drives unidirectional progression of insect metamorphosis.

Although the above E75 decay model nicely explains $\beta F T Z$-F1 induction in peripheral tissues, it does not rule out the possibility that NOS-mediated E75 inactivation is also involved in this process. Interestingly, 20E has been shown to repress NOS activity via a nongenomic pathway in another insect species (Champlin and Truman 2000). It is conceivable, therefore, that NOS activity is suppressed during the 20E peak, allowing E75 to inhibit $\beta F T Z-F 1$ transcription. Upon decline of the 20E titer, NOS activity is restored and NO prevents the inhibitory activity of residual E75, reinforcing DHR3-mediated $\beta F T Z-F 1$ expression (Fig. 1). It is worth noting in this context that NOS activity is known to be required for the proper progression of metamorphic growth of adult structures. When NOS activity is suppressed before initiation of metamorphosis, proper coordination of cell growth and differentiation is disrupted, resulting in the enlargement of some appendages (Kuzin et al. 1996). Interestingly, the hypertrophic adult leg segments observed in these animals are similar to the malformed leg structures in $\beta F T Z$-F1 mutants (Broadus et al. 1999; Rewitz et al. 2010). Therefore, as discussed by Cáceres et al. (2011), NOS is likely to be involved in the regulation of $\beta F T Z-F 1$ expression in many tissues, highlighting its central role as an initiator and mediator of insect metamorphosis. 


\section{What is regulating NOS expression and function?}

Having discovered that NOS plays a critical role in the initiation of insect metamorphosis, it will be important to determine what regulates its expression and function, both in the PG as well as the peripheral tissues. Considering the critical role of 20E in the regulation of NOS/ E75/DHR3 expression and function in peripheral tissues, one might expect that their expression and activity in the PG could be regulated in a feedback manner by $20 \mathrm{E}$. However, using a "ligand trap" reporter system, a previous study showed that the PG does not respond to hemolymph 20E fluctuations like other tissues (Kozlova and Thummel 2002). Indeed, the PG expression profiles of E75, DHR3, and some other 20E-inducible nuclear receptors (KingJones et al. 2005; Cáceres et al. 2011) seem to be clearly different from their overall expression profiles in the whole body (King-Jones and Thummel 2005), suggesting that the PG response to $20 \mathrm{E}$ production is programmed differently than peripheral tissues. Unveiling the underlying molecular mechanisms that regulate the expression of NOS, E75, and DHR3 in the PG is an important next step for determining how ecdysteroidogenesis is regulated in the course of insect development.

With respect to the regulation of NOS activity, several cofactors such as $\mathrm{Ca}^{2+} /$ calmodulin are required for full enzymatic function (Muller 1994; Regulski and Tully 1995). In Malpighian (renal) tubules of Drosophila, two neuropeptides, capa- 1 and capa-2, increase fluid secretion rates through the up-regulation of intracellular $\mathrm{Ca}^{2+}$ concentration and subsequent NOS/cyclic GMP signaling (Kean et al. 2002). Since $\mathrm{Ca}^{2+}$ signaling is known to have an important role in the fly PG (Venkatesh and Hasan 1997) and some neuropeptides like the prothoracicotropic hormone (PTTH) are known to activate the $\mathrm{Ca}^{2+}$ signaling pathway in the PG to regulate ecdysteroidogenesis (Gilbert et al. 2002), it will be interesting to see whether the activities of these ecdysteroidogenic neuropeptides are mediated by NOS.

Other factors such as oxygen supply can also affect NOS function. Despite the fact that oxygen is an indispensable substrate for NOS-mediated production of NO, low oxygen supply (hypoxia) is known to induce NO signaling (Wingrove and O'Farrell 1999; Dijkers and O'Farrell 2009) rather than suppressing it. This is possibly through the reduction of nitrite in the mitochondria, which serves as a pool of NO produced by NOS during normoxia. This interesting role of $\mathrm{NO}$ as a signaling component of hypoxia deserves more attention when considering the close link between hypoxia and insect body size (Kaiser et al. 2007). Since the timing of the onset of metamorphosis is one of the major determinants of insect body size (Edgar 2006), NOS-mediated initiation of metamorphosis through $\beta$ FTZ-F1 might provide an important link between body size and oxygen level.

\section{NO-E75 interaction-beyond regulation of $\beta F T Z-F 1$ expression}

As discussed above, Cáceres et al. (2011) successfully demonstrated that the NO-E75 interaction plays a key role in regulating $\beta F T Z$-F1 expression and thereby initiating metamorphosis. But some results of this study also indicate that the NO-E75 interaction likely goes further than simply regulating $\beta F T Z$ - $F 1$ expression. When a constitutively active NOS isoform was overexpressed in the PG, Cáceres et al. (2011) observed that larval growth was arrested at an earlier stage: The larvae did not pass beyond the second instar. It is unlikely that this earlier stage growth arrest results from ectopic expression of $\beta F T Z-F 1$ in the PG, since up-regulation of $\beta F T Z-F 1$ should accelerate the production of ecdysone and thereby promote developmental progression. Indeed, Cáceres et al. (2011) could partially rescue the developmental defect of NOS-overexpressed larvae by ecdysone feeding, indicating that the phenotype is also caused by the loss of ecdysone and not overproduction. Importantly, a similar phenotype was observed when E75 expression was suppressed in the PG (Cáceres et al. 2011). Together with their epistasis analysis, it is clear that the NOS overexpression phenotype is mediated by the suppression of E75 function. This is also consistent with the mutant phenotype of one E75 isoform, where the majority of mutant individuals also fail to progress into the third instar because of low ecdysteroid titer (Bialecki et al. 2002). Therefore, apart from its role as a suppressor of $\beta F T Z$ - $F 1$ expression, E75 seems to have one or more positive effects on ecdysteroidogenesis at earlier developmental stages, which is again suppressed by NOS/NO. Whether these additional roles of NO-E75 interaction are mediated by any other DHR3 target genes (Vorobyeva et al. 2011) remains to be elucidated.

Cáceres et al. (2011) also observed one other phenotype that might be independent of $\beta F T Z-F 1$ expression: an enormous increase in PG cell size associated with loss of NOS. Although the function of NO in regulation of cell proliferation has been well investigated (Kuzin et al. 1996; Villalobo 2006), its effect on cell size has been poorly documented. It might be notable here that DHR3 has recently been shown to have an unexpected role as a regulator of cell-autonomous growth through S6 kinase (Montagne et al. 2010). Alternatively, in the absence of NOS, PTTH might continue to signal through the Torso/ERK pathway (Rewitz et al. 2009), leading to extra endoreplication cycles in the PG cells (Ghosh et al. 2010). Double knockdown of various components in the PG-for example, NOS and torso-will help establish the epistatic relationship of these various signaling pathways that contribute to PG size control.

In summary, the study by Cáceres et al. (2011) provides a solid example of direct NO-mediated transcriptional regulation in vivo, and we can expect that many new developmental processes that use this system will be discovered both in insects and vertebrates in the near future. Such findings will broaden our understanding of NO biology and how it contributes to important biological processes, such as the coordination of developmental timing, metabolism, and behavior.

\section{Acknowledgments}

N.Y. is supported by a post-doctoral fellowship from the Japan Society for the Promotion of Science, and M.B.O. by RO1 GM095746. 


\section{References}

Berreur P, Porcheron P, Moriniere M, Berreur-Bonnenfant J, Belinski-Deutsch S, Busson D, Lamour-Audit C. 1984. Ecdysteroids during the third larval instar in 1 (3)ecd-1ts, a temperature-sensitive mutant of Drosophila melanogaster. Gen Comp Endocrinol 54: 76-84.

Bialecki M, Shilton A, Fichtenberg C, Segraves WA, Thummel CS. 2002. Loss of the ecdysteroid-inducible E75A orphan nuclear receptor uncouples molting from metamorphosis in Drosophila. Dev Cell 3: 209-220.

Broadus J, McCabe JR, Endrizzi B, Thummel CS, Woodard CT. 1999. The Drosophila $\beta F T Z-F 1$ orphan nuclear receptor provides competence for stage-specific responses to the steroid hormone ecdysone. Mol Cell 3: 143-149.

Cáceres L, Necakov AS, Schwartz C, Kimber S, Roberts IJH, Krause HM. 2011. Nitric oxide coordinates metabolism, growth, and development via the nuclear receptor E75. Genes Dev (this issue). doi: 10.1101/gad.2064111.

Champlin DT, Truman JW. 2000. Ecdysteroid coordinates optic lobe neurogenesis via a nitric oxide signaling pathway. Development 127: 3543-3551.

Dijkers PF, O'Farrell PH. 2009. Dissection of a hypoxia-induced, nitric oxide-mediated signaling cascade. Mol Biol Cell 20: 4083-4090.

Edgar BA. 2006. How flies get their size: genetics meets physiology. Nat Rev Genet 7: 907-916.

Foley E, O'Farrell PH. 2003. Nitric oxide contributes to induction of innate immune responses to Gram-negative bacteria in Drosophila. Genes Dev 17: 115-125.

Ghosh A, McBrayer Z, O'Connor MB. 2010. The Drosophila gap gene giant regulates ecdysone production through specification of the PTTH-producing neurons. Dev Biol 347: 271278.

Gibbens YY, Warren JT, Gilbert LI, O'Connor MB. 2011. Neuroendocrine regulation of Drosophila metamorphosis requires TGF $\beta /$ Activin signaling. Development 138: 26932703.

Gibbs SM, Truman JW. 1998. Nitric oxide and cyclic GMP regulate retinal patterning in the optic lobe of Drosophila. Neuron 20: 83-93.

Gilbert LI, Rybczynski R, Warren JT. 2002. Control and biochemical nature of the ecdysteroidogenic pathway. Annu Rev Entomol 47: 883-916.

Hess DT, Matsumoto A, Kim SO, Marshall HE, Stamler JS. 2005. Protein S-nitrosylation: purview and parameters. Nat Rev Mol Cell Biol 6: 150-166.

Horner MA, Chen T, Thummel CS. 1995. Ecdysteroid regulation and DNA binding properties of Drosophila nuclear hormone receptor superfamily members. Dev Biol 168: 490-502.

Kageyama Y, Masuda S, Hirose S, Ueda H. 1997. Temporal regulation of the mid-prepupal gene FTZ-F1: DHR3 early late gene product is one of the plural positive regulators. Genes Cells 2: 559-569.

Kaiser A, Klok CJ, Socha JJ, Lee WK, Quinlan MC, Harrison JF. 2007. Increase in tracheal investment with beetle size supports hypothesis of oxygen limitation on insect gigantism. Proc Natl Acad Sci 104: 13198-13203.

Kean L, Cazenave W, Costes L, Broderick KE, Graham S, Pollock VP, Davies SA, Veenstra JA, Dow JA. 2002. Two nitridergic peptides are encoded by the gene capability in Drosophila melanogaster. Am I Physiol Regul Integr Comp Physiol 282: R1297-R1307. doi: 10.1152/ajpregu.00584.2001.

King-Jones K, Thummel CS. 2005. Nuclear receptors-a perspective from Drosophila. Nat Rev Genet 6: 311-323.
King-Jones K, Charles JP, Lam G, Thummel CS. 2005. The ecdysone-induced DHR4 orphan nuclear receptor coordinates growth and maturation in Drosophila. Cell 121: 773784.

Kozlova T, Thummel CS. 2002. Spatial patterns of ecdysteroid receptor activation during the onset of Drosophila metamorphosis. Development 129: 1739-1750.

Kuzin B, Roberts I, Peunova N, Enikolopov G. 1996. Nitric oxide regulates cell proliferation during Drosophila development. Cell 87: 639-649.

Lam GT, Jiang C, Thummel CS. 1997. Coordination of larval and prepupal gene expression by the DHR3 orphan receptor during Drosophila metamorphosis. Development 124: 17571769.

Marvin KA, Reinking JL, Lee AJ, Pardee K, Krause HM, Burstyn JN. 2009. Nuclear receptors homo sapiens Reverb $\beta$ and Drosophila melanogaster E75 are thiolate-ligated heme proteins which undergo redox-mediated ligand switching and bind CO and NO. Biochemistry 48: 70567071 .

Montagne J, Lecerf C, Parvy JP, Bennion JM, Radimerski T, Ruhf ML, Zilbermann F, Vouilloz N, Stocker H, Hafen E, et al. 2010. The nuclear receptor DHR3 modulates dS6 kinasedependent growth in Drosophila. PLoS Genet 6: e1000937. doi: 10.1371/journal.pgen.1000937.

Muller U. 1994. $\mathrm{Ca}^{2+} /$ calmodulin-dependent nitric oxide synthase in Apis mellifera and Drosophila melanogaster. Eur J Neurosci 6: 1362-1370.

Parvy JP, Blais C, Bernard F, Warren JT, Petryk A, Gilbert LI, O'Connor MB, Dauphin-Villemant C. 2005. A role for $\beta F T Z$ F1 in regulating ecdysteroid titers during post-embryonic development in Drosophila melanogaster. Dev Biol 282: 84-94.

Regulski M, Tully T. 1995. Molecular and biochemical characterization of dNOS: a Drosophila $\mathrm{Ca}^{2+} /$ calmodulindependent nitric oxide synthase. Proc Natl Acad Sci 92: 9072-9076.

Regulski M, Stasiv Y, Tully T, Enikolopov G. 2004. Essential function of nitric oxide synthase in Drosophila. Curr Biol 14: R881-R882. doi: 10.1016/j.cub.2004.09.068.

Reinking J, Lam MM, Pardee K, Sampson HM, Liu S, Yang P, Williams S, White W, Lajoie G, Edwards A, et al. 2005. The Drosophila nuclear receptor E75 contains heme and is gas responsive. Cell 122: 195-207.

Rewitz KF, Yamanaka N, Gilbert LI, O'Connor MB. 2009. The insect neuropeptide PTTH activates receptor tyrosine kinase torso to initiate metamorphosis. Science 326: 14031405.

Rewitz KF, Yamanaka N, O'Connor MB. 2010. Steroid hormone inactivation is required during the juvenile-adult transition in Drosophila. Dev Cell 19: 895-902.

Segraves WA, Hogness DS. 1990. The E75 ecdysone-inducible gene responsible for the 75B early puff in Drosophila encodes two new members of the steroid receptor superfamily. Genes Dev 4: 204-219.

Venkatesh K, Hasan G. 1997. Disruption of the IP3 receptor gene of Drosophila affects larval metamorphosis and ecdysone release. Curr Biol 7: 500-509.

Villalobo A. 2006. Nitric oxide and cell proliferation. FEBS J 273: 2329-2344.

Vorobyeva NE, Nikolenko JV, Krasnov AN, Kuzmina JL, Panov VV, Nabirochkina EN, Georgieva SG, Shidlovskii YV. 2011. SAYP interacts with DHR3 nuclear receptor and participates in ecdysone-dependent transcription regulation. Cell Cycle 10: $1821-1827$. 
White KP, Hurban P, Watanabe T, Hogness DS. 1997. Coordination of Drosophila metamorphosis by two ecdysone-induced nuclear receptors. Science 276: 114-117.

Wingrove JA, O'Farrell PH. 1999. Nitric oxide contributes to behavioral, cellular, and developmental responses to low oxygen in Drosophila. Cell 98: 105-114.

Woodard CT, Baehrecke EH, Thummel CS. 1994. A molecular mechanism for the stage specificity of the Drosophila prepupal genetic response to ecdysone. Cell 79: 607-615.

Yakubovich N, Silva EA, O'Farrell PH. 2010. Nitric oxide synthase is not essential for Drosophila development. Curr Biol 20: R141-R142. doi: 10.1016/j.cub.2009.12.011.

Yamada M, Murata T, Hirose S, Lavorgna G, Suzuki E, Ueda H. 2000. Temporally restricted expression of transcription factor $\beta F T Z-F 1$ : significance for embryogenesis, molting and metamorphosis in Drosophila melanogaster. Development 127: 5083-5092. 


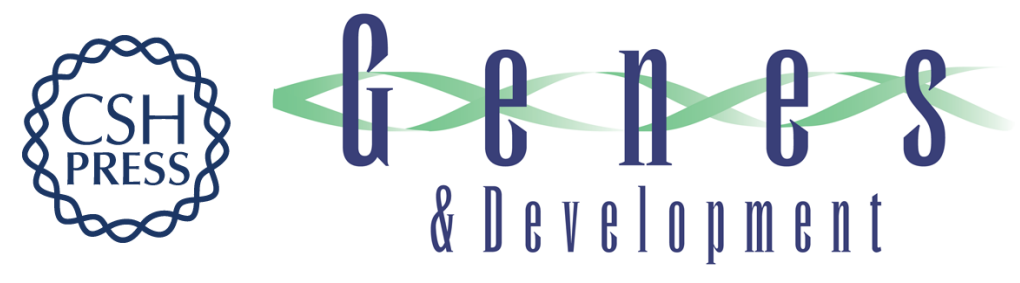

\section{Nitric oxide directly regulates gene expression during Drosophila development: need some gas to drive into metamorphosis?}

Naoki Yamanaka and Michael B. O'Connor

Genes Dev. 2011, 25:

Access the most recent version at doi:10.1101/gad.2080411

Related Content Nitric oxide coordinates metabolism, growth, and development via the nuclear receptor E75

Lucía Cáceres, Aleksandar S. Necakov, Carol Schwartz, et al.

Genes Dev. July , 2011 25: 1476-1485

References This article cites 39 articles, 12 of which can be accessed free at:

http://genesdev.cshlp.org/content/25/14/1459.full.html\#ref-list-1

Articles cited in:

http://genesdev.cshlp.org/content/25/14/1459.full.html\#related-urls

License

Email Alerting Receive free email alerts when new articles cite this article - sign up in the box at the top Service right corner of the article or click here.

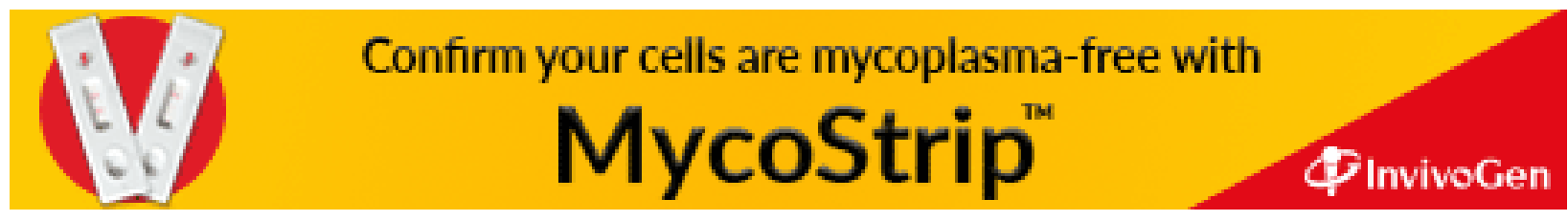

